\title{
Detection of salient objects with focused attention based on spatial and temporal coherence
}

\author{
WU Yang ${ }^{1 *}$, ZHENG NanNing $^{1 *}$, YUAN ZeJian $^{1}$, JIANG HuaiZu $^{1} \&$ LIU Tie ${ }^{2}$ \\ ${ }^{1}$ Institute of Artificial Intelligence and Robotics, Xi'an Jiaotong University, Xi'an 710049, China; \\ ${ }^{2}$ IBM Research-China, Beijing 100193, China
}

Received October 8, 2010; accepted November 8, 2010

\begin{abstract}
The understanding and analysis of video content are fundamentally important for numerous applications, including video summarization, retrieval, navigation, and editing. An important part of this process is to detect salient (which usually means important and interesting) objects in video segments. Unlike existing approaches, we propose a method that combines the saliency measurement with spatial and temporal coherence. The integration of spatial and temporal coherence is inspired by the focused attention in human vision. In the proposed method, the spatial coherence of low-level visual grouping cues (e.g. appearance and motion) helps per-frame object-background separation, while the temporal coherence of the object properties (e.g. shape and appearance) ensures consistent object localization over time, and thus the method is robust to unexpected environment changes and camera vibrations. Having developed an efficient optimization strategy based on coarse-to-fine multi-scale dynamic programming, we evaluate our method using a challenging dataset that is freely available together with this paper. We show the effectiveness and complementariness of the two types of coherence, and demonstrate that they can significantly improve the performance of salient object detection in videos.
\end{abstract}

visual attention, focused attention, salient object detection, spatial and temporal coherence

Citation: Wu Y, Zheng N N, Yuan Z J, et al. Detection of salient objects with focused attention based on spatial and temporal coherence. Chinese Sci Bull, 2011, 56: 1055-1062, doi: 10.1007/s11434-011-4387-1

The rapid development of networks and storage devices has greatly encouraged the capture, spread and sharing of large quantities of video data. However, because these extensive videos often contain unimportant or uninteresting content, searching for a desired video segment in a large database becomes a very difficult and time-consuming task. To solve this problem, a plausible approach is to model visual attention of human vision, as it can filter out unwanted information in a scene [1].

In the literature, visual attention has been widely modeled as a visual saliency estimation problem [2-5], which has been extensively investigated using both static images and video segments. Instead of disregarding the content when dealing with the generic visual saliency estimation

*Corresponding authors (email: wuyang0321@gmail.com; nnzheng@mail.xjtu.edu.cn) problem in video [5], we focus on detecting a salient object in video segments. Video segments with a particular focus are found in many kinds of videos, and research on these has very important applications such as video summarization, retrieval, navigation and editing.

As argued by cognitive scientist Stephen Palmer in his book [6], there are two types of visual attention: distributed attention and focused attention. The former relates to visual signal processing that occurs when subjects are prepared for the target to appear in any location, while the latter occurs when they have selected a single perceptual object. For the problem of detecting a salient object in a video clip, focused attention of the human vision system should play a critical role. Therefore, the perceptive properties of focused attention become our inspiration for solving the problem.

Most of the related works focus on the integration of 
various bottom-up cues for estimating saliency [7-9], supported by feature integration theory [10]. Usually such cues are represented by contrast-based features, including both low-level measures of contrast (e.g. pixel-wise color, gradient contrast and regional appearance contrast) and highlevel measures (e.g. objectness [11]). When human eyefixation records or hand-labeled bounding boxes of salient regions are available for model learning [3-5], these top-down priors can be used to train a saliency model, whose properties depend largely on the training data. An extreme case is to train class-specific saliency estimators that can be used to detect interesting object classes [12]. There are also some studies on the combination of bottom-up and top-down models for better detection performance [5,13]. Irrespective of which cues or priors have been used, most of these efforts are aimed at generating a saliency map, without explicitly modeling the coherence of the results, either spatially or temporally. Although spatial and temporal information can somehow be represented by regional contrast, we argue that coherence is not equivalent to regional contrast, because it emphasizes the absolute consistency of the object itself, and not the relative differences against the changing background.

Spatial coherence has been used widely in image and motion segmentation [14,15], while temporal coherence plays a critical role in object tracking [16]. Though both of these have also been used for moving object detection $[17,18]$, they are usually modeled separately. As far as we are aware, there is no prior work that uses both of these factors to detect salient objects in videos. However, when humans focus their attention on an object, it means that the object remains somewhere consistently in the spatial and temporal space. Therefore, spatial and temporal coherence are indispensable properties for detecting the object.

This paper proposes a novel method that combines spatial and temporal coherence systematically with the saliency measurement for salient object detection. We present encouraging results that demonstrate the importance and complementariness of these two types of coherence.

\section{Problem formulation}

We formulate salient object detection as a binary labeling problem, where the salient object is represented as a binary mask $A_{t}=\left\{a_{\mathrm{x}}^{t}\right\}$ for each frame $I_{t}, t \in\{1, \cdots, T\}$ in a video segment $\boldsymbol{I}=I_{1: T}$. In the mask, $a_{\mathbf{x}}^{t} \in\{0,1\}$ is a binary label for each pixel $\mathbf{x}$ in frame $I_{t}$ and $T$ is the number of frames in the video segment. More concretely, $a_{\mathrm{x}}^{t}=1$ indicates that pixel $\mathrm{x}$ belongs to the salient object, while $a_{\mathrm{x}}^{t}=0$ means the opposite. Following the widely used conditional random field model [19], the probability of the prediction $\boldsymbol{A}=A_{1: T}$ can be modeled as a conditional distribution:

$$
P(\boldsymbol{A} \mid \boldsymbol{I})=P\left(A_{1: T} \mid I_{1: T}\right)=\frac{1}{Z} \exp \left(-E\left(A_{1: T} \mid I_{1: T}\right),\right.
$$

where $E(\cdot)$ is an energy function and $Z$ is the partition function. Therefore, the optimal solution for the labeling $A_{1: T}$ based on the maximum a posteriori estimation can be obtained by minimizing $E\left(A_{1: T} \mid I_{1: T}\right)$. If the Markov property holds for the data, the optimization can be written as:

$$
\boldsymbol{A}^{*}=\arg \min _{A_{t: T}} E\left(A_{1: T} \mid I_{1: T}\right)=\arg \min _{A_{t: T}} \sum_{t} E\left(A_{t} \mid I_{t, t-1}, A_{t-1}\right) .
$$

The energy function of the binary mask of each frame $E\left(A_{t} \mid I_{t, t-1}, A_{t-1}\right)$ can further be decomposed into the following three terms:

$$
\begin{aligned}
E\left(A_{t} \mid I_{t, t-1}, A_{t-1}\right)= & \underbrace{S\left(A_{t}, I_{t}, M_{t}\right)}_{\text {Saliency }}+\alpha \underbrace{C_{S}\left(A_{t}, I_{t}, M_{t}\right)}_{\text {Spatial coherence }} \\
& +\beta \underbrace{C_{T}\left(A_{t-1}, A_{t}, I_{t-1}, I_{t}\right)}_{\text {Temporal coherence }},
\end{aligned}
$$

where $M_{t}$ is the optical flow field from frame $I_{t-1}$ to frame $I_{t}$, while $\alpha$ and $\beta$ are the balancing weights for the spatial and temporal coherence, respectively.

Note that eq. (3) is the general form of the global energy function based on both the static image and dynamic motion information, where the optical flow field $M_{t}$ and the saliency map $S\left(A_{t}, I_{t}, M_{t}\right)$ can be obtained using any method. In fact, these computations are currently two active research topics $[5,20]$ with new approaches constantly being proposed despite the existence of many solutions.

In our former work [4], we proposed the three different types of features defined below, to compute the saliency maps of static images.

(i) Local multi-scale contrast. Given an image $I$, a Gaussian image pyramid of $L$ levels is computed, and then the multi-scale contrast feature is defined as ${ }^{1)}$

$$
f_{c}(\mathbf{x}, I) \propto \sum_{l=1}^{L} \sum_{\mathbf{x}^{\prime} \in \mathcal{N}(\mathbf{x})}\left\|I^{l}(\mathbf{x})-I^{l}\left(\mathbf{x}^{\prime}\right)\right\|^{2},
$$

where $I^{l}$ is the $l$ th level image in the pyramid and $\mathcal{N}(\mathbf{x})$ is the neighborhood of pixel $\mathbf{x}$. Typically, $L=6$ and $\mathcal{N}(\mathbf{x})$ is a $9 \times 9$ window.

(ii) Regional center-surround histogram. Suppose the salient object is bounded by a rectangle $R$, we construct a surrounding contour $R_{S}$ with the same area as $R$. Given a pixel $\mathbf{x}$, we find the most distinct rectangle $R^{*}(\mathbf{x})$ centered at $\mathbf{x}$, from the candidates with different sizes and aspect ratios:

$$
R^{*}(\mathbf{x})=\arg \max _{R(\mathbf{x})} \chi^{2}\left(R(\mathbf{x}), R_{S}(\mathbf{x})\right) .
$$


The size of $R$ varies within $[0.1,0.7] \times \min (w, h)$, where $w, h$ are the image width and height, respectively. The aspect ratio is one of the five values $\{0.5,0.75,1.0,1.5,2.0\}$. The $\chi^{2}$ distance measures the dissimilarity of the two RGB color histograms of the center and surrounding rectangles. The center-surround histogram feature is defined as a weighted sum of the $\chi^{2}$ distance at neighboring pixels:

$$
f_{h}(\mathbf{x}, I) \propto \sum_{\left\{\mathbf{x}^{\prime} \mid \mathbf{x} \in R^{*}\left(\mathbf{x}^{\prime}\right)\right\}} w_{\mathbf{x x}} \chi^{2}\left(R^{*}\left(\mathbf{x}^{\prime}\right), R_{S}^{*}\left(\mathbf{x}^{\prime}\right)\right),
$$

where $w_{\mathbf{x x}^{\prime}}=\exp \left(-0.5 \sigma_{\mathbf{x}^{\prime}}^{-2}\left\|\mathbf{x}-\mathbf{x}^{\prime}\right\|^{2}\right)$ is a Gaussian falloff weight with variance $\sigma_{\mathbf{x}^{\prime}}^{2}$ set to one third of the size of $R^{*}\left(\mathbf{x}^{\prime}\right)$, making the weight adaptive to the data.

(iii) Global color spatial-distribution. This feature measures the spatial variance of the color. Suppose the colors in the image can be represented by a Gaussian mixture model:

$$
p\left(c \mid I_{\mathbf{x}}\right)=\frac{w_{c} N\left(I_{\mathbf{x}} \mid \mu_{c}, \Sigma_{c}\right)}{\sum_{c=1}^{C} w_{c} N\left(I_{\mathbf{x}} \mid \mu_{c}, \Sigma_{c}\right)},
$$

where $C$ is the number of color components. Then, after obtaining the position variance $V(c)$ of each color component $c$, the color spatial-distribution feature can be defined as

$$
f_{s}(\mathbf{x}, I) \propto \sum_{c} p\left(c \mid I_{\mathbf{x}}\right) \cdot(1-V(c)) .
$$

Further details about the definition and computation of $V(c)$ can be found in [4].

These features have been extensively evaluated, with promising results, using a large-scale image dataset. The integrated saliency map based thereon is

$$
F_{S_{t}}(\mathbf{x})=\lambda_{c} f_{c}(\mathbf{x}, I)+\lambda_{h} f_{h}(\mathbf{x}, I)+\lambda_{s} f_{s}(\mathbf{x}, I),
$$

where the learned optimal parameters are $\lambda_{c}=0.24, \lambda_{h}=0.54$, and $\lambda_{s}=0.22$ [4].

Static saliency may be confused by a cluttered background especially in videos, so we have also proposed computing the same features on the optical flow fields to represent the dynamic motion saliency [21]. However, because of the instability of the optical flow extraction algorithm, the optical flow field was smoothed before feature extraction. If the optical flow is computed by a satisfactory approach such as the one proposed in [22], the features can be extracted directly from $M_{t}$ without extra smoothing. In the experiments presented in Section 4, optical flow is computed by the algorithm introduced in [22], and the same parameters $\left(\lambda_{c}, \lambda_{h}\right.$ and $\left.\lambda_{s}\right)$ are adopted to combine the three types of features into the motion saliency map $F_{M_{t}}(\mathbf{x})$.

Having obtained both these saliency maps, we can combine them into an overall saliency map $F_{t}$ using the adaptive strategy proposed in [21]. Then, the saliency term in eq. (3) can be defined as

$$
S\left(A_{t}, I_{t}, M_{t}\right)=\sum_{\mathbf{x}: a_{\mathbf{x}}^{t}=0} F_{t}\left(\mathbf{x}, I_{t}, M_{t}\right)+\sum_{\mathbf{x}: a_{\mathbf{x}}^{t}=1}\left(1-F_{t}\left(\mathbf{x}, I_{t}, M_{t}\right)\right) .
$$

Figure 1 illustrates the framework of our method based on eq. (3). Besides the saliency maps, spatial and temporal coherence are two other important components for global optimization, as explained in detail in the following section.

\section{Spatial and temporal coherence}

\subsection{Spatial coherence}

The spatial relationship between neighboring pixels has widely been used to represent the intrinsic property of random fields. The simplest relationship is based on the
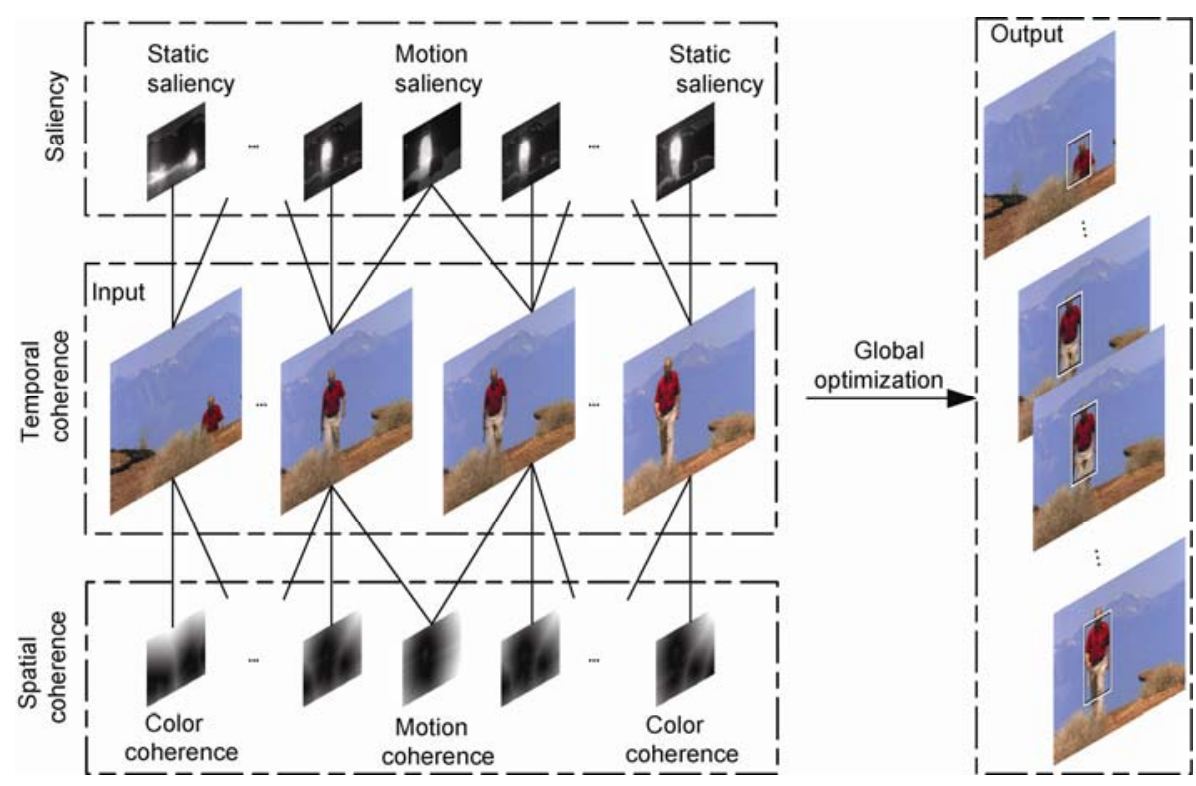

Figure 1 Overall framework of our method. 
assumption that proximal pixels are more likely to have the same category labels. In conditional random field-based applications, however, information from the data is usually taken into account as well, resulting in a more general spatial constraint because neighboring pixels belonging to the same category are more likely to share the same features/properties. Such a spatial coherence constraint is also widely referred to as the smoothing term or smoothing model in the literature because it tends to smoothen the features/properties within the same category. Consequently, we formulate the spatial coherence as

$$
C_{S}\left(A_{t}, I_{t}, M_{t}\right)=\sum_{\mathbf{x}, \mathbf{x}^{\prime}} \delta\left(a_{\mathbf{x}^{\prime}}^{t} \neq a_{\mathbf{x}}^{t}\right) D\left(\mathbf{x}, I_{t}, M_{t}\right),
$$

where $\mathbf{x}$ and $\mathbf{x}^{\prime}$ are neighboring pixels and $D$ is the smoothness measurement of the image and motion fields at pixel $\mathbf{x}$. The smoother the image and motion fields are at $\mathbf{x}$, the larger the value of $D\left(\mathbf{x}, I_{t}, M_{t}\right)$ is. Therefore, when $C_{S}$ is minimized, the boundary pixels separating the foreground object and the background regions tend to be located at or close to the edge points of the image and motion field. A simple yet effective choice for $D$ is the distance transform [23], which is a soft smoothness measurement. If chamfer distance is used as the distance metric, it can be computed efficiently.

The formulation above is a generic form that contains both the static appearance and dynamic motion information. Here, we discuss these two typical cues, together with the combination thereof.

(i) Static cue: appearance. Although in general the static cue can be any image features, we focus on the most widely used one: raw color information. If only this cue is considered, eq. (11) becomes

$$
C_{S}\left(A_{t}, I_{t}\right)=\sum_{\mathbf{x}, \mathbf{x}^{\prime}} \delta\left(a_{\mathbf{x}^{\prime}}^{t} \neq a_{\mathbf{x}}^{t}\right) D_{c h}\left(\mathbf{x}, E M\left(I_{t}\right)\right),
$$

where $\operatorname{EM}\left(I_{t}\right)$ is the edge map of $I_{t}$ and $D_{c h}$ is the chamfer distance.

The static cue uses the image edges to guide the labeling, which should be beneficial for the results if the background is relatively clean. Unfortunately, there are many cases in which the background is cluttered, especially outdoor scenes as shown in Figure 2, where row (d) illustrates the

(a)

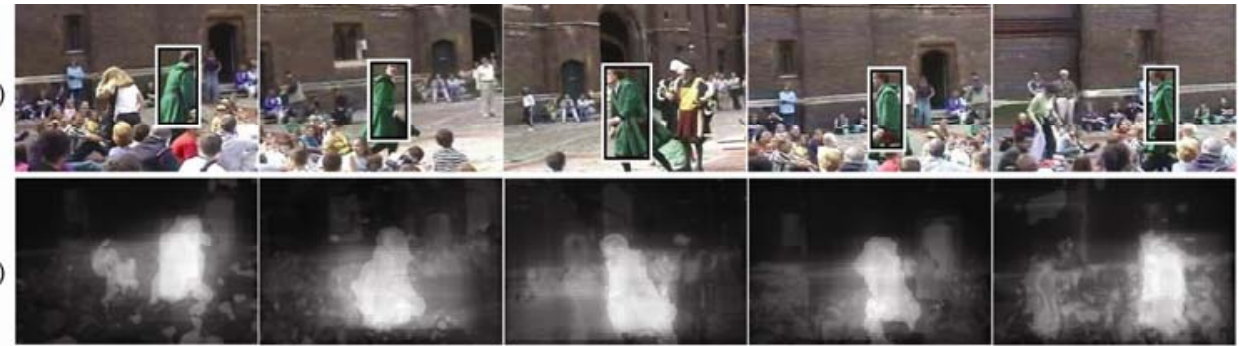

(c)

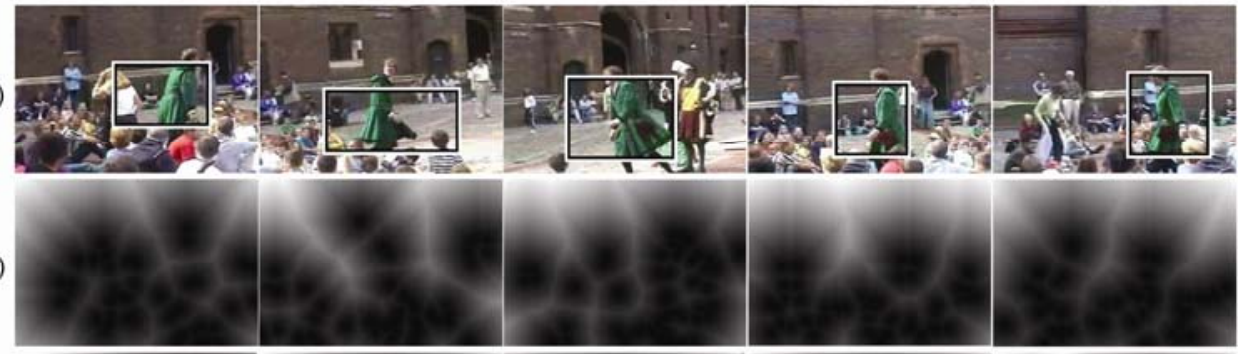

(e)

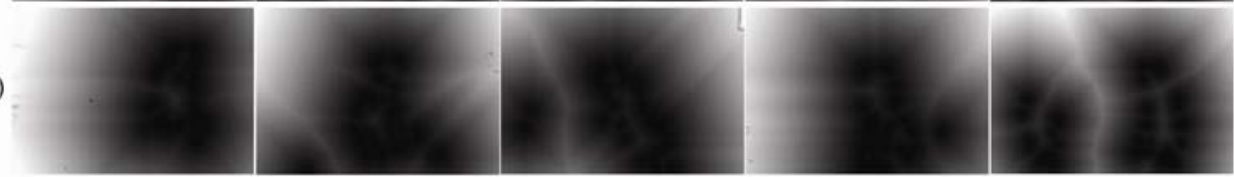

(f)

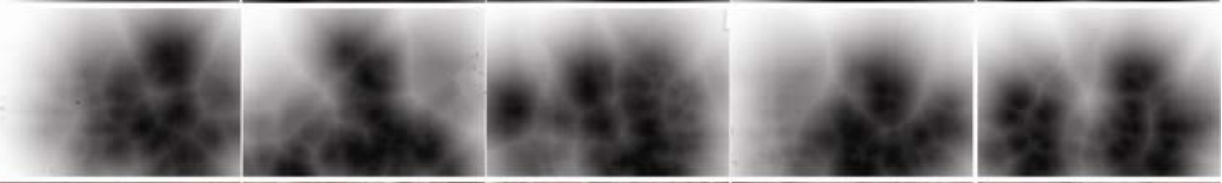

(g)

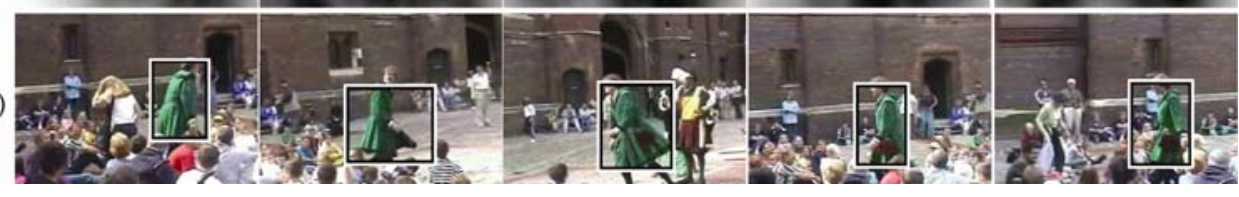

Figure 2 Effectiveness of spatial coherence. (a) Sample video frames with ground truth labels; (b) saliency maps; (c) results based on saliency maps only; (d), (e), and (f) distance transform maps of the spatial coherence based on color cue, motion cue, and both color and motion cues, respectively, where the darkest areas (valleys) are potential object boundaries; (g) detection results using both saliency and spatial coherence. 
distance transform results using the static cue only. The valleys (darkest areas) are spread out across the whole images because of the cluttered background. In these cases, optimization using appearance-based spatial coherence can easily get confused because it cannot distinguish the real object boundaries from the background clutter.

(ii) Dynamic cue: motion. If the foreground object moves differently to the cluttered background, the optical flow field $M_{t}$ is useful for detecting the object. Similar to the appearance-based spatial coherence, we can define spatial coherence of the motion field as

$$
C_{S}\left(A_{t}, M_{t}\right)=\sum_{\mathbf{x}, \mathbf{x}^{\prime}} \delta\left(a_{\mathbf{x}^{\prime}}^{t} \neq a_{\mathbf{x}}^{t}\right) D_{c h}\left(\mathbf{x}, E M\left(M_{t}\right)\right),
$$

where $\operatorname{EM}\left(M_{t}\right)$ is the edge map of $M_{t}$ and $D_{c h}$ is once again the chamfer distance. As far as we are aware, this is the first time that the optical flow field has been used as the spatial coherence constraint to detect salient objects in videos. The first 4 frames of row (e) in Figure 2 demonstrate the effectiveness of spatial coherence when the cluttered background moves uniformly yet differently to the salient object.

(iii) Integrating static and dynamic cues. When the salient object moves in a dynamic scene with an uneven background motion field, the power of the dynamic cue is weakened. In these cases, the static cue may be helpful if the moving parts of the background have weaker static boundaries than the salient objects. Therefore, combining the two cues might be a better choice than using only one of them. Inspired by the way in which the spatial and temporal smoothing terms are combined for moving object detection in reference [24], we combined the two cues for spatial coherence by defining the smoothness measurement $D\left(\mathbf{x}, I_{t}, M_{t}\right)$ in eq. (11) as

$$
\begin{aligned}
D\left(\mathbf{x}, I_{t}, M_{t}\right)= & 1-\left[1-\eta_{I} \cdot D_{c h}\left(\mathbf{x}, E M\left(I_{t}\right)\right)\right] \\
& \times\left[1-\eta_{M} \cdot D_{c h}\left(\mathbf{x}, E M\left(M_{t}\right)\right)\right],
\end{aligned}
$$

where $\eta_{I} \in[0,1]$ and $\eta_{M} \in[0,1]$ are two parameters controlling the influence of the static and dynamic cues, respectively (where 0 means ineffective and 1 means fully involved). Such a combination can reduce the risk of being confused by the clutter or movement in the background. The last two rows in Figure 2 show the combined distance transform map (i.e. $D\left(\mathbf{x}, I_{t}, M_{t}\right)$ at each pixel) and the detection results using the two-cue based spatial coherence, for which $\eta_{I}$ and $\eta_{M}$ are both set to 1 . It can be seen that the results are much tighter than those using only saliency maps.

\subsection{Temporal coherence}

Temporal coherence is designed to represent the temporal similarity between the salient objects from two consecutive frames. Such temporal similarity can be measured by both shape and appearance as

$$
\begin{aligned}
C_{T}\left(A_{t-1, t}, I_{t-1, t}\right) & =\beta_{S} C_{T}^{\text {Shape }}\left(A_{t-1}, A_{t}\right) \\
& +C_{T}^{\text {Appearance }}\left(A_{t-1, t}, I_{t-1, t}\right), \\
C_{T}^{\text {Shape }}\left(A_{t-1}, A_{t}\right) & =\sum_{\mathbf{x}}\left|a_{\mathbf{x}}^{t-1}-a_{\mathbf{x}}^{t}\right|, \\
C_{T}^{\text {Appearance }}\left(A_{t-1, t}, I_{t-1, t}\right) & =\chi^{2}\left(H\left(A_{t-1}, I_{t-1}\right), H\left(A_{t}, I_{t}\right)\right),
\end{aligned}
$$

where $H(A, I)$ is the appearance histogram of area $A$ in frame $I$ (in our experiments, only color has been used as the appearance representation). In practice, we find that setting the balancing weight to $\beta_{S}=\frac{0.5 \times w \times h}{w+h} \times 100$ works well with $w, h$ denoting the image width and height, respectively.

Figure 3 shows an example of how temporal coherence works. The five consecutive frames are prone to a short and irregular vibration of the camera, causing the saliency maps to be heavily influenced by the distracting motion. However, the temporal coherence can smooth out the randomness within the saliency maps and generate a stable sequence of object bounding boxes.

\section{Optimization strategy}

Given the problem in eqs. (2) and (3), the optimization involves finding the best labeling for each pixel $\mathbf{x}$ in each frame $I_{t}$ of the video segment $\mathbf{I}=I_{1: T}$. This is computationally infeasible as the search space is $2^{w h T}$, where $w, h$ denote the image width and height, respectively. In object detection, however, pixel-wise segmentation results are usually unnecessary and the bounding boxes suffice. If the binary mask $A_{t}$ is restricted to a rectangle, it can be represented by four parameters: center $\mathbf{x}_{A}^{t}$ and size $\mathbf{s}_{A}^{t}$, both of which are two-dimensional. The state space thus decreases dramatically to no more than $\left(w^{2} \times h^{2}\right)^{T}$. Meanwhile, the temporal coherence based on shape can be simplified as

$$
C_{T}^{\text {Shape }}\left(A_{t-1}, A_{t}\right)=\left\|\mathbf{x}_{A}^{t}-\mathbf{x}_{A}^{t-1}\right\|^{2}+\gamma\left\|\mathbf{s}_{A}^{t}-\mathbf{s}_{A}^{t-1}\right\|^{2},
$$

where $\gamma$ is a weight balancing the location and scale differences according to [25].

The problem defined by eqs. (2) and (3) can be decomposed into a set of subproblems, the solutions of which enable the original optimization problem to be solved quickly. Moreover, these subproblems overlap, i.e., their local solutions can be used multiple times in the global optimization. Therefore, the optimization can be solved efficiently by dynamic programming.

Let $B_{t}\left(A_{t}\right)$ be the summarized energy of frames 1 to $t$, then these subproblems can be written recursively as 
(a)

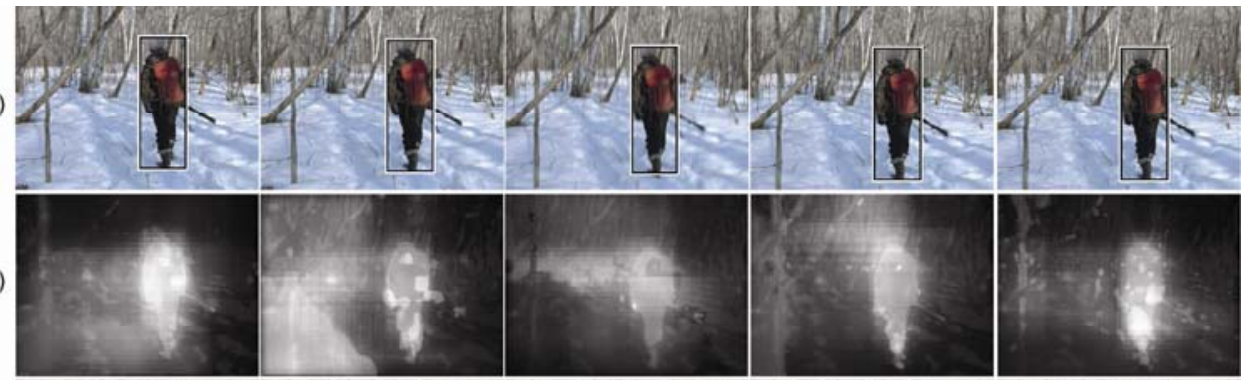

(c)

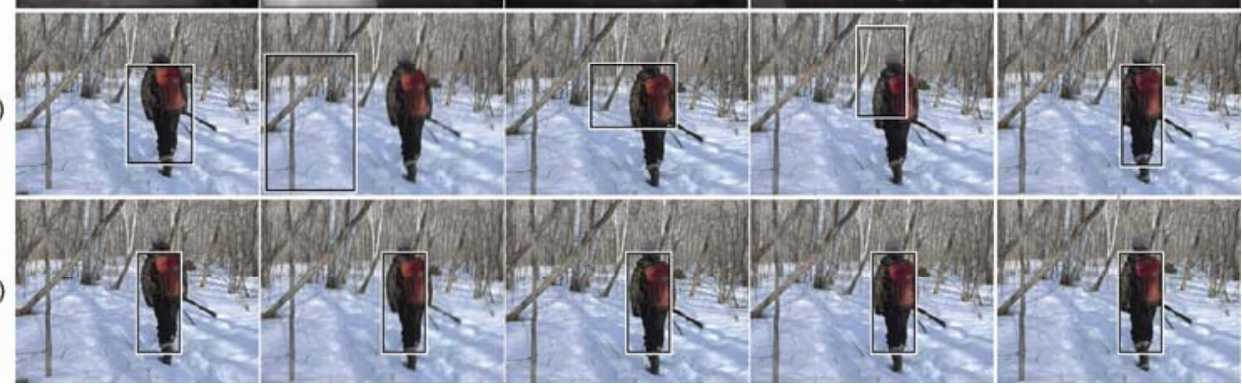

Figure 3 Effectiveness of temporal coherence. (a) Sample video frames with ground truth labels; (b) saliency maps; (c) results using saliency maps only; (d) results using both saliency and temporal coherence.

$$
\begin{aligned}
B_{1}\left(A_{1}\right)= & E\left(A_{1} \mid I_{1: T}\right)=S\left(A_{1}, I_{1}\right)+\alpha C_{S}\left(A_{1}, I_{1}\right), \\
B_{t}\left(A_{t}\right)= & \min _{A_{t-1} \in \mathcal{N}_{D P}\left(A_{t}\right)}\left\{B_{t-1}\left(A_{t-1}\right)+E\left(A_{t} \mid I_{t, t-1}, A_{t-1}\right)\right\} \\
= & S\left(A_{t}, I_{t}, M_{t}\right)+\alpha C_{S}\left(A_{t}, I_{t}, M_{t}\right) \\
& +\min _{A_{t-1} \in \mathcal{N}_{D P}\left(A_{t}\right)}\left(B_{t-1}\left(A_{t-1}\right)+\beta C_{T}\left(A_{t-1, t}, I_{t-1, t}\right)\right) .
\end{aligned}
$$

Note that we narrow down the search space of $A_{t-1}$ by enforcing it to be in the neighborhood of $A_{t}$, which coincides with the temporal coherence requirement.

After computing all possible $B_{t}\left(A_{t}\right)$ for each frame $I_{t}$, the final solution for the overall problem can be obtained by

$$
A_{T}^{*}=\arg \min _{A_{T}} B_{T}\left(A_{T}\right) .
$$

Then we trace back the sequence to obtain the optimal solution for each of the rest frames $A_{t}, t \in\{1, \cdots, T-1\}$ :

$$
A_{t}^{*}=\arg \min _{A_{t} \in \mathcal{N}_{D P}\left(A_{t+1}^{*}\right)}\left(B_{t}\left(A_{t}\right)+\beta C_{T}\left(A_{t}, A_{t+1}^{*}, I_{t, t+1}\right)\right) .
$$

Suppose the number of possible $A_{t}$ in $I_{t}$ is $N$ (no more than $w^{2} h^{2}$ ), and $N T$ is not very large, the index of $A_{t}^{*}$ can be cached to a table of every possible $A_{t+1}$, with size $N T$. Then the trace back operation can be done in $O(T)$ time by merely consulting the lookup table $A_{t}^{*}=T\left(A_{t+1}^{*}\right)$.

The computational expense of the algorithm is dominated by the computation of $B_{t}\left(A_{t}\right), t \in\{1, \cdots, T\}$. Suppose there are $n$ candidates in the neighborhood space $\mathcal{N}_{D P}\left(A_{t}\right)$, then the time cost is $O(n N T)$. Because of the variations in object size, it is not easy to set a proper neighborhood size $n$. If $n$ is too small, it may fail to represent the large movements of big objects, whereas conversely, an overlarge $n$ loses the efficiency of the neighborhood search. To solve this problem and further speed up the computation, a coarse-to-fine strategy can be used [21]. All the saliency maps are down-sampled to generate a pyramid (for example 6 layers) and then, after searching the entire state space at the coarsest scale, the optimization results serve as the initial solution for the finer scale. By doing this, the neighbor space can be limited to a small range, such as a circle with a radius of 2 pixels.

\section{Experimental results}

To demonstrate quantitatively the advantages of using spatial and temporal coherence, we carried out our experiments on 32 video segments with a total of 4820 frames collected from the internet ${ }^{2)}$. Each video segment contains a single salient object, which ranges from humans performing various activities and animals in the wild, to vehicles both on the ground and in the air. All the frames are annotated with object bounding boxes, and the detection performance is evaluated in terms of mean precision $(\mathrm{P})$, recall $(\mathrm{R})$, F-measure $\left(F_{0.5}\right)$, and boundary displacement errors (BDE) [3].

Four different settings on eq. (3) have been adopted for performance comparison: saliency only, namely setting $\alpha=$ $\beta=0$; involving spatial coherence (Saliency $+\mathrm{SC}$ ), i.e. $\beta=$ 0 ; involving temporal coherence (Saliency + TC), i.e.

2) This dataset is freely available from the authors. 
$\alpha=0$; and involving both spatial and temporal coherence (Saliency + STC). For spatial coherence, we chose $\alpha=0.125$ because other values such as $0.025,0.05,0.25,0.75$ gave poorer results, while $\eta_{I}$ and $\eta_{M}$ were set to 1 because smaller values resulted in lower performance gain, and we found that using both cues was better than using either of them. For the temporal coherence, $\gamma=2$ and $\beta=0.01$ were found to be effective. The mean performances of the four different settings, illustrated in Figure 4, provide several valuable results.

(i) Spatial coherence increases the precision significantly, but at the same time diminishes the recall somewhat. Overall, it improves the performance in terms of both the F-measure and BDE. Moreover, adding SC to "Saliency" yielded a $10.9 \%$ increase in precision and a $3.8 \%$ decrease in recall, whereas adding SC to "Saliency + TC" improved precision by $14.3 \%$ and reduced recall by $7.1 \%$. The performance gains with respect to the F-measure (BDE) for these two cases are $6.2 \%(10.3 \%)$ and $7.0 \%(10.1 \%)$, respectively.

(ii) Temporal coherence increases both the precision and the recall and therefore, significantly improves the F-mea- sure and BDE. Moreover, adding TC to "Saliency" improved precision by $9.2 \%$ and recall by $3.8 \%$, whereas adding TC to "Saliency + SC" improved precision by $12.6 \%$ and recall by $0.3 \%$. The performance improvements in terms of F-measure (BDE) for these two cases are $7.6 \%$ $(23.6 \%)$ and $8.3 \%(23.4 \%)$, respectively.

(iii) Involving both SC and TC is better than using only one of them, and can greatly improve the detection performance. Compared with "Saliency", "Saliency + STC" improved the F-measure by $15.1 \%$ and reduced BDE by $31.3 \%$.

Figure 5 shows two representative object detection results using these four different strategies. The benefits of involving spatial and temporal coherence can be clearly seen. The experimental results show that spatial coherence and temporal coherence are in fact complementary. The existence of either of them has no significant impact on the properties of the other. Although in our model the spatial coherence includes the between-frame motion, it only requires the motion field within the object to be as consistent as possible, whereas temporal coherence constrains the magnitude of the motion vectors.
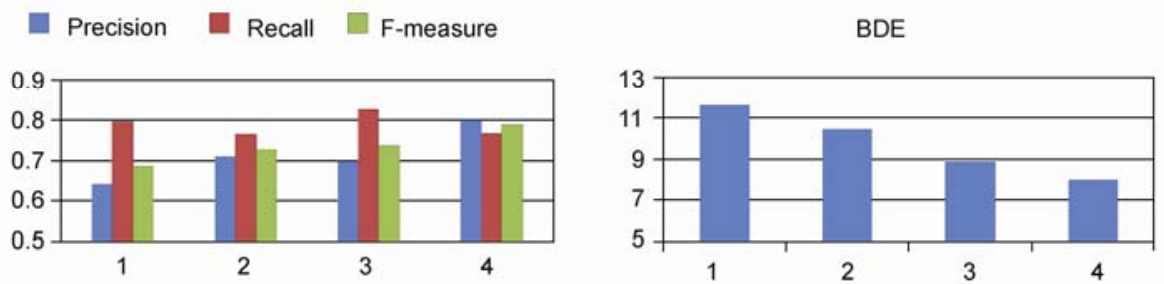

Figure 4 Mean performance comparison for evaluating the effectiveness of spatial and temporal coherence. The four different settings on the horizontal axis are: 1 , no coherence (saliency only); 2 , involving spatial coherence; 3 , involving temporal coherence; 4 , involving both spatial and temporal coherence.

(a)

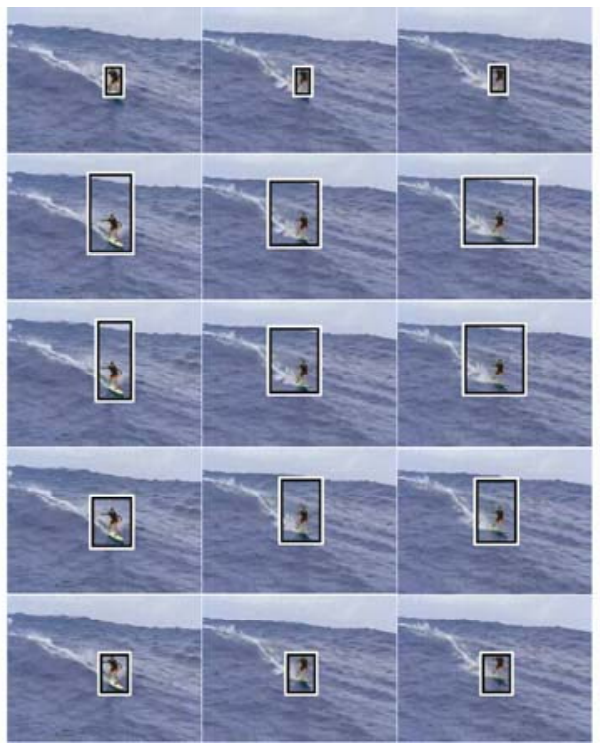

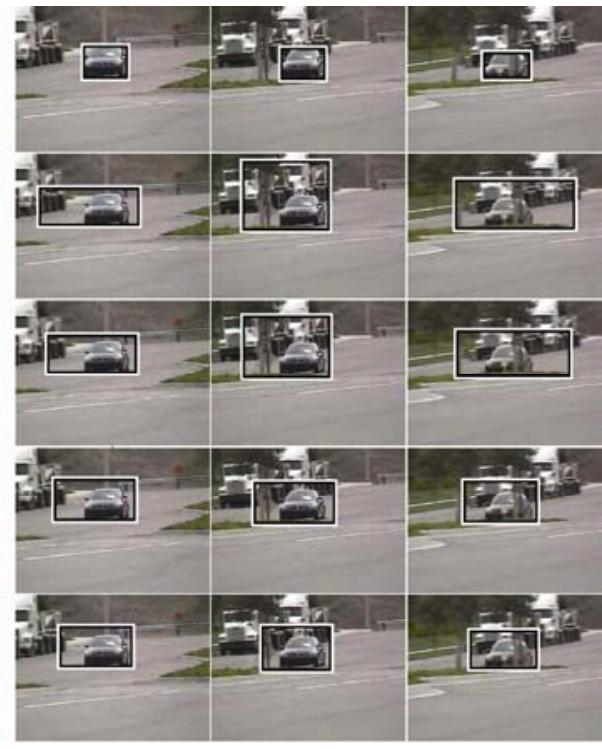

Figure 5 Qualitative results using sampled video frames. (a) Input video segments with ground truth labels; (b), (c), (d) and (e) detection results using the four different strategies: Saliency, Saliency + SC, Saliency + TC and Saliency + STC, respectively. 


\section{Conclusion and discussion}

Inspired by the focused attention inherent in human vision, we proposed a novel method for salient object detection that incorporates both spatial and temporal coherence with traditional saliency measurements. Experimental results demonstrate the effectiveness and complementariness of the spatial and temporal coherence of saliency features. The method is simple and flexible in the sense that any saliency computation models can be directly embedded therein, and new cues on modeling the within-frame spatial coherence and between-frame temporal coherence can easily be added. Moreover, dynamic programming and a coarse-to-fine search strategy have been used to solve the problem efficiently.

The limitation of this work is that it assumes that the salient object appears throughout the whole video segment, which implicitly requires the segment to be extracted in advance from a longer video. A possible future work is to detect when the focused attention start and end, so that the video segment can be localized automatically.

This work was supported by the National Natural Science Foundation of China (60635050 and 90820017) and the National Basic Research Program of China (2007CB311005).

1 Itti L, Rees G, Tsotsos J. Neurobiology of Attention. San Diego: Elsevier, 2005

2 Treue S. Visual attention: The where, what, how and why of saliency. Curr Opin Neurobiol, 2003, 13: 428-432

3 Liu T, Sun J, Zheng N N, et al. Learning to detect a salient object. In: Proceedings of IEEE Computer Society Conference on Computer and Vision Pattern Recognition, Minneapolis, USA, 2007. 1-8

4 Liu T, Yuan Z J, Sun J, et al. Learning to detect a salient object. IEEE Trans Pattern Anal Mach Intell, 02 Mar. 2010, doi: 10.1109/TPAMI. 2010.70

5 Li J, Tian Y H, Huang T J, et al. Probabilistic multi-task learning for visual saliency estimation in video. Int J Comput Vis, 2010, 90: 150-165

6 Palmer S. Vision Science: Photons to Phenomenology. Cambridge, MA: The MIT Press, 1999

7 Itti L, Koch C, Niebur E. A model of saliency-based visual attention for rapid scene analysis. IEEE Trans Pattern Anal Mach Intell, 1998, 20: 1254-1259

8 Itti L, Baldi P. A principled approach to detecting surprising events in video. In: Proceedings of the IEEE Computer Society Conference on Computer and Vision Pattern Recognition, San Diego, CA, USA, 2005. 631-637

9 Walther D, Koch C. Modeling attention to salient protoobjects. Neu- ral Netw, 2006, 19: 1395-1407

10 Treisman A M, Gelade G. A feature-integration theory of attention. Cogn Psychol, 1980, 12: 97-136

11 Alexe B, Deselaers T, Ferrari V. What is an object? In: Proceedings of IEEE Computer Society Conference on Computer and Vision Pattern Recognition, San Francisco, USA, 2010. 1-8

12 Moosmann F, Larlus D, Jurie F. Learning saliency maps for object categorization. In: Proceedings of ECCV International Workshop on the Representation and Use of Prior Knowledge in Vision, Graz, Austria, 2006. 1-14

13 Peters R J, Itti L. Beyond bottom-up: incorporating Task dependent influences into a computational model of spatial attention. In: Proceedings of IEEE Computer Society Conference on Computer and Vision Pattern Recognition, Minneapolis, USA, 2007. 1-8

14 Cao L L, Li F F. Spatially coherent latent topic model for concurrent segmentation and classification of objects and scenes. In: Proceedings of IEEE International Conference on Computer Vision, Rio de Janeiro, Brazil, 2007. 1-8

15 Weiss Y, Adelson E H. A unified mixture framework for motion segmentation: incorporating spatial coherence and estimating the number of models. In: Proceedings of IEEE Computer Society Conference on Computer and Vision Pattern Recognition, San Francisco, USA, 1996. 321-326

16 Moscheni F, Dufaux F, Kunt M. Object tracking based on temporal and spatial information. In: Proceedings of IEEE Conference on Acoustics, Speech, and Signal Processing, Atlanta, USA, 1996. 1914-1917

17 Kim M, Choi J G, Kim D, et al. A VOP generation tool: Automatic segmentation of moving objects in image sequences based on spatio-temporal information. IEEE Trans Circuits Syst Video Technol, 1999, 9: 1216-1226

18 Tsaig Y, Averbuch A. Automatic segmentation of moving objects in video sequences: A region labeling approach. IEEE Trans Circuits Syst Video Technol, 2002, 12: 597-612

19 Lafferty J, McCallum A, Pereira F. Conditional random fields: Probabilistic models for segmenting and labeling sequence data. In: Brodley C E, Danyluk A P, eds. Proceedings of International Conference on Machine Learning, Williams College, Williamstown, MA, USA, 2001, 282-289

20 Baker S, Roth S, Scharstein D, et al. A database and evaluation methodology for optical flow. In: Proceedings of IEEE International Conference on Computer Vision, Rio de Janeiro, Brazil, 2007. 1-8

21 Liu T, Zheng N N, Ding W, et al. Video attention: Learning to detect a salient object sequence. In: Proceedings of International Conference on Pattern Recognition, Tampa, Florida, USA, 2008. 1-4

22 Brox T, Bruhn A, Papenberg N, et al. High accuracy optical flow estimation based on a theory for warping. In: Pajdla T, Matas J, eds. Proceedings of European Conference on Computer Vision, Prague, Czech Republic, 2004. 25-36

23 Rosenfeld A, Pfaltz J. Sequential operations in digital picture processing. J ACM, 1966, 13: 471-494

24 Yang H, Tian J, Chu Y, et al. Spatiotemporal smooth models for moving object detection. IEEE Signal Process Lett, 2008, 15: 497-500

25 Sun J, Zhang W, Tang X, et al. Bidirectional tracking using trajectory segment analysis. In: Proceedings of IEEE International Conference on Computer Vision, Beijing, China, 2005. 717-724

Open Access This article is distributed under the terms of the Creative Commons Attribution License which permits any use, distribution, and reproduction in any medium, provided the original author(s) and source are credited. 